\title{
OTROS TRABAJOS
}

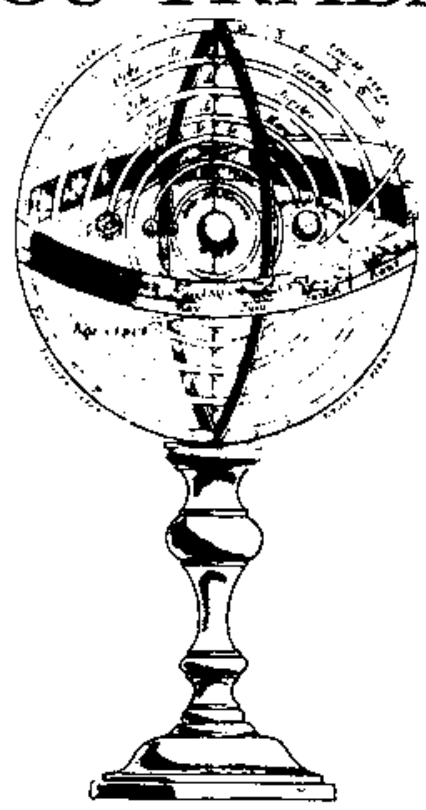

\section{BASES DE DATOS DE CIENCIAS DE LA EDUCACION EN EL MUNDO}

ANTA CABREROS, C. y MaTa bILbao, A.

Instituto de Información y Documentación en Ciencias Sociales y Humanidades. 1SOC. (CSIC)

\section{SUMMARY}

The purpose of this paper is to let the professionals of research and education know the most relevant data processors in the area of Educational Sciences, showing their different characteristics, name, editor, scope, size, issues and sources they implement.

Para conocer las publicaciones existentes en un área del conocimiento, es imprescindible actualmente acudir a las técnicas de documentación científica automatizadas, que nos brindan la oportunidad de conseguir esta información o tener noticia de su existencia de una forma ágil y rápida, ya que con los medios tradicionales - todavía importantes actualmente- y con el crecimiento exponencial de las publicaciones científicas, sería excesivo el tiempo necesario para conocer su existencia o tener referencia de su contenido.
Por otra parte, la fuerte implantación del sector de la industria de la información a partir de los años 70 , es* pecialmente en el mundo anglosajón, y cada vez más en el resto de Europa, nos facilita este acceso.

En 1983 el Ministerio de Educación y Ciencia, tomó la iniciativa de estudiar las posibilidades físicas y lógicas que tenía su Centro de Proceso de Datos para la creación y distribución de bases de datos documentales. Se concluyó con cierta rapidez que el soporte físi- 
co existente - un ordenador UNIVAC $1100 / 80-$ y el soporte lógico tolerable por el mismo -el Sistema UNIDAS - de recuperación de la información podian resultar válidos para los objetivos perseguidos. Inmediatamente, se inició la creación de las bases de datos documentales que posteriormente describiremos.

En la actualidad de las 2.400 bases de datos que FUIN. CA reseña en su libro «LAS BASES DE DATOS DEL MUNDO), 26 pertenecen al área de Ciencias de la Educación, de ellas 15 son estadounidenses, 12 europeas y de estas últimas $S$ son españolas. El Instituto de información y Documentación en Ciencias Sociales y Humanidades (ISOC), del Consejo Superior de Investiga. ciones Cientificas, además de crear la base de datos "ISOC" de Ciencias Sociales y Humanidades, hace de intermediario entre los usuarios que lo solicitan y las bases de datos españolas e internacionales en la recuperación de la información bibliográfica y en la con. secución de los documentos primarios.

El objetivo de este articulo es dar a conocer a los profesionales de la investigación y de la enseñanza, las bases de datos más significativas en el panorama mundial en el area de Ciencias de la Educación con sus distintas caracteristicas, nombre, productor, cobertura, tamaño, temática y fuentes que recogen.

\section{BASES DE DATOS ESPAÑOLAS EN CIEN- CIAS DE LA EDUCACION}

\subsection{Base "ISOC"}

Productor: Instituto de Formación y Documentación en Ciencias Sociales y Humanidades (ISOC) del Consejo Superior de Investigaciones Científicas; es una base de datos bibliográfica que contiene las referencias de los artículos aparecidos en las Revistas Españolas desde 1975, con la siguiente cobertura temática: Ciencias de la Educación, Psicología, Sociología, Economia, Ciencias Políticas, Urbanismo, Documentación Científica, Administración Pública, Derecho, Historia, Geografía, Filología, Literatura, Arqueología, Filoso. fia, Filología, Religión y Bellas Artes.

Fuentes: Recoge revistas españolas editadas por Unjversidades, Reales Academias, Institutos del CSIC, Ins" tituciones religiosas, Diputaciones, Entidades locales, etc. indicándose de! orden de 438 títulos de los que más de 50 pertenecen a Clencias de la Educación.

El lenguaje de recuperación es semicontrolado, se excluyen polisemias y sinonimias y se hacen referencias cruzadas a los términos más significativos. Los elementos de información de cada documento se estructuran en siete campos recuperables:

Autor, Institución donde trabaja el autor, título del ar. tículo, revista (fecha de publicación, ISSN, localización del documento, tipo de documento y modo informatjvo, clasificación temática, número de referencias bibliográficas) notas y descriptores.

42
También se editan de forma impresa los índices que contienen las referencias bibliográficas, con periodicidad anual en las siguientes series:

- Serie A - Psicología y Ciencias de la Educación.

- Serie B - Economía, Sociología, Ciencias Políticas y Urbanismo.

- Serie C - Derecho.

- Serie D - Ciencia y Documentación Científica e ín. dice Espantol de Humanidades.

\subsection{Base DEDALO}

Productor: Centro de Información y Documentación Educativa (CIDE), del Ministerio de Educación y Ciencia; es una base de datos bibliográfica que pretende recoger toda la documentación educativa, -libros, re. vistas, informes y otros documentos existentes en los fondos de las bibliotecas que dependen del Ministerio de Educación y Ciencia.

\subsection{Base BIDE}

Productor: Oficina de Educación Iberoamericana; la Base de Datos BIDE (Banco Iberoamericano de Datos sobre Educación), es una base de datos informativa, que recoge las convocatorias de Cursos y Becas de todo el mundo a los que pueden acceder estudiantes iberoamericanos, facilita información suministrada por: Universidades, Instituciones y Estados que convocan los mencionados cursos y becas, está en funcionamiento desde 1984 y su actualización se produce a medida que recibe la información de dichas convocatorias, los elementos de información son:

Cursos. Ente Organizador, Sede del Curso, Número de Plazas, Idioma en el que se desarrolla, Duración, Materias, Requisitos que deben reunir los participantes, Título que se otorga, Lugar de Inscripción, Becas inherentes al curso.

Becas. Organismo que la concede, Características, Número disponible, Materias para las que se concede, $\mathrm{Re}_{\text {- }}$ quisitos que deben reunir los solicitantes, Duración de la Beca, Dotación de la Beca, Lugar de solicitud.

\subsection{Base LEDA}

Productor: Centro de Proceso de Datos del Ministerio de Educación y Ciencia. Recoge la legislación sobre educación de carácter general promulgada por el Estado o por las Comunidades Autonómicas con competencias en materia de educación.

Fuentes: Boletines y Diarios Oficiales del Estado y las Comunidades autónomas.

ESTRUCTURA DE LA BASE Y ELEMENTOS DE INFORMACION. - LEDA relaciona una serie de documentos cuya recuperación permite al usuario un conocimiento exacto y completo de la normativa general en materia de educación, vigente o derogada, asi como las convocatorias de pruebas, concursos, subven- 
ciones, cursos, oposiciones, becas y ayudas al estudio, en los siguientes tesauros.

Tesauro 1 "LEDA VIGENTE» relaciona la legislación educativa de carácter general, emanada del Estado y de las Comunidades Autónomas con competencias en materia de educación, que haya sido publicada en los correspondientes Diarios o Boletines Oficiales. En es. te tesauro no aparecen las diversas convocatorias de concursos, oposiciones, subvenciones, ayudas, pruebas, etc. que corresponden a! Tesauro 4. Tampoco los actos administrativos, tales como creaciones, supresiones, modificaciones de centros, nombramientos, etc.

Tesauro 2 «LEDA DEROGADA», relaciona la legislación no vigente, entendiendo por tal la expresamente derogada o la que, teniendo una vigencia limitada, ha cumplido el plazo de vigencia predeterminado. Las derogaciones implícitas no son tenidas en cuenta.

Tesauro 3 "LEDA REFERIDA» permite recuperar todos los documéntos relacionados con una disposición concreta, es decir, todas las normas que hayan citado a dicha disposición o que hayan sido citadas por ella expresamente.

Tesauro 4 "LEDA CONVOCA» incluye las convoca torias de concursos, oposiciones, concursosoposiciones, concursos de traslados, cursos, subvenciones, ayudas, pruebas, etc. realizadas por el Estado o por las Comunidades Autonómicas con competencias en materia educativa, publicadas desde el 1 de Enero de 1985 . En este caso el documento no reproduce el texto íntegro de las disposiciones sino solamente el título.

Cada uno de los documentos se sistematiza en las siguientes partes: aspectos, descriptores, título de la dis* posición, indices, parte informativa y texto integro de la norma.

\subsection{Base REDINED}

1 Productor: Centro de Información y Documentación Educativa (CIDE) del Ministerio de Educación y Ciencia y las Consejerias de Educación de cada una de las Comunidades Autónomas.

La base REDINED, Red Estatal de Bases de Datos sobre Investigaciones Educativas; recoge las tesis, tesinas y proyectos de investigación en educación producidos por la Comunidad Científica Española.

\section{ACCESO A LAS BASES Y RECUPERA- CION DE LA INFORMACION}

Estas bases son distribuidas por Centro de Proceso de Datos del Ministerio de Educación y Ciencia, de modo interactivo a través de la red telefónica conmutada desde cualquier punto, tanto a rivel nacional como internacional y próximamente lo será a través de la red de transmisión de datos IBERPAC.

ENSEÑANZA DE LAS CIENCIAS, 1987, $S$ (1)
El sistema de recuperación es UNIDAS, que permite realizar la búsqueda de la información hasta en un máximo de 10 tesauros simultáneos a través de:

—Aspectos significativos del documento.

- Descriptores o palabras clave.

- Texto libre.

También es preciso señalar las Bases creadas y distribuidas por el Ministerio de Cultura, Puntos de Información Cultural (PIC). Este Servicio PIC, es un servicio público que se ofrece a los interesados a través de sedes instaladas en dependencias del Ministerio de Curtura y de entidades colaboradoras, en más de 40 ciudades españolas. Los usuarios pueden dirigirse personalmente a alguna de estas sedes o por correo al Programa PIC del Ministerio de Cultura para efectuar consuitas.

Las Bases Bibliográficas que distribuye son: Bibliografía Española; Bibliografia Especializada: Administración Pública, Ciencias de la Información, Ciencia y Tecnologia, Publicidad; Bibliografía Extranjera depositada en la Biblioteca Nacional; Registros Sonoros depositados en la Biblioteca Nacional; Censo de Bibliotecas; Catálogo Colectivo de Publicaciones Periódicas; Censo de Editoriales; Hemeroteca Nacional; Libros editados en España.

\section{BASES DE DATOS INTERNACIONALES DE CIENCIAS DE LA EDUCACION}

En el Cuadro I se presenta un resumen de las principales bases de datos internacionales de la educación, indicando nombre, productor, temática, fuentes, tamaño y cobertura temporal.

\section{REFERENCIAS BIBLIOGRAFICAS}

ANTA CABREROS, C. y SAN MILLAN BUJANDA, ${ }^{2}$ J., 1985, Base de datos bibliográfica ISOC, Comunicación presentada en el I Congreso Iberoamericano de Informática y Documentación en Medelín (Colombia).

SECRETARIA GENERAL TECNICA. CENTRO DE PROCESO DE DATOS, Bases de datos documentales. Guía del usuario, Ministerio de Educación y Ciencia.

FUINCA, Bases de datos del mundo. 
Cuadro 1

\begin{tabular}{|c|c|c|c|c|c|}
\hline Notere & Focouras & Tawica & FEMTES & zemento & MEmpm \\
\hline 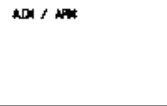 & 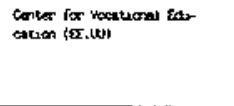 & 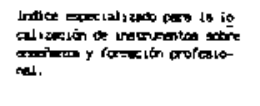 & 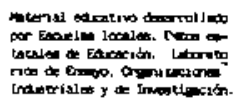 & 17.500 & $1607.15 \%$ \\
\hline 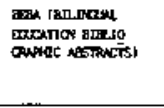 & 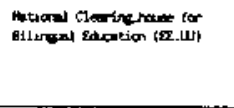 & 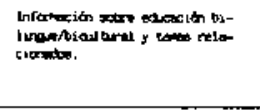 & 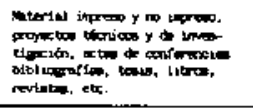 & 15.000 & $\lim _{1 \rightarrow \infty}$ \\
\hline RTRO & 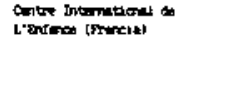 & 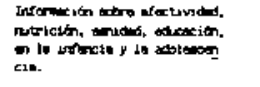 & 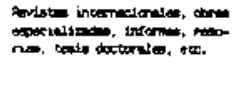 & $\infty 2.000$ & 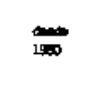 \\
\hline 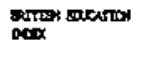 & 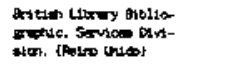 & 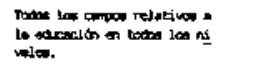 & Aurto of toraciton & 30.000 & $\underset{1900}{\longrightarrow}$ \\
\hline 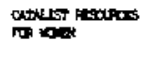 & 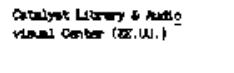 & 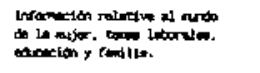 & 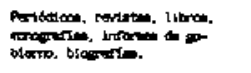 & 4,000 & 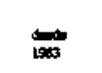 \\
\hline 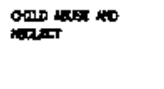 & 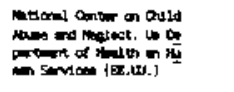 & 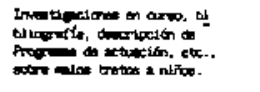 & 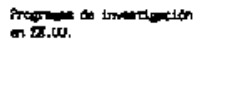 & 12.400 & im \\
\hline 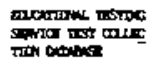 & 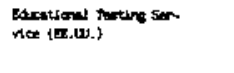 & 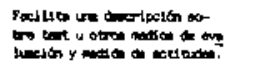 & $\cdots$ & 5.000 & $\cdots$ \\
\hline ance & 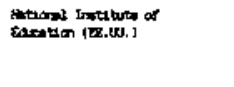 & 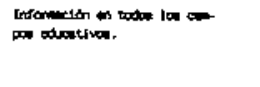 & 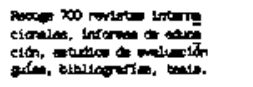 & 90.00 & to \\
\hline 0000 & 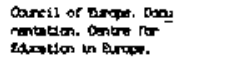 & 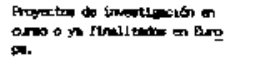 & 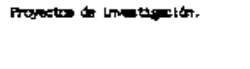 & $0.2=0$ & is/5 \\
\hline 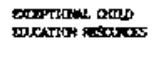 & 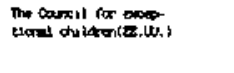 & 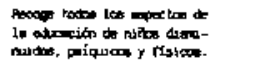 & 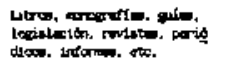 & 52.000 & 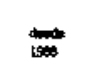 \\
\hline 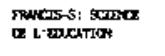 & Contres thention on iv. & 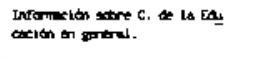 & 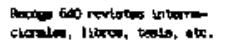 & 76.000 & thre \\
\hline eepess & 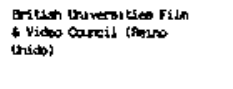 & 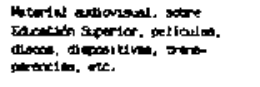 & n. & 5.50 & $x a=3$ \\
\hline Des & 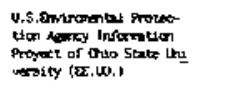 & 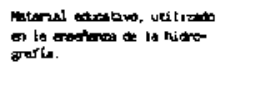 & 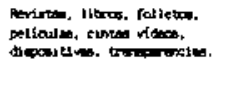 & 6.100 & ${ }_{19 \pi}^{\infty}$ \\
\hline music & 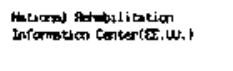 & 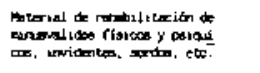 & 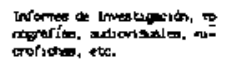 & 3.00 & $\underset{1900}{\infty}$ \\
\hline NICOX & 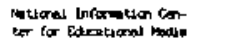 & 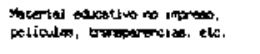 & 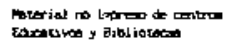 & 391.150 & $\infty_{1900}^{\infty}$ \\
\hline 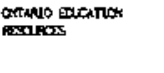 & 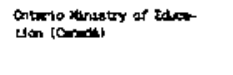 & 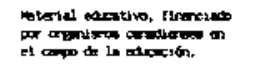 & - - - & $6 . \infty$ & $\underset{1900}{\infty 1900}$ \\
\hline 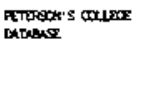 & 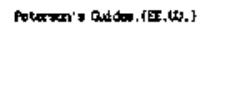 & 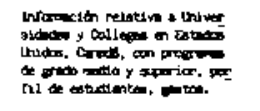 & - - - - - - & $3 . \infty 0$ & soves \\
\hline $\operatorname{sic}$ & 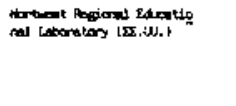 & 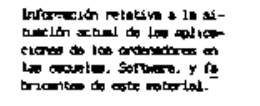 & $\ldots$ & 2.000 & 要网 \\
\hline 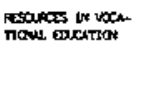 & 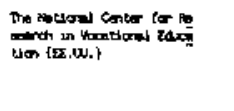 & 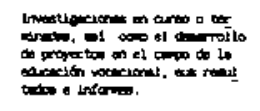 & - . - n & 4.300 & $\underset{b \infty \pi}{\infty}$ \\
\hline 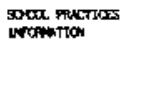 & 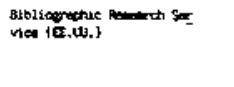 & 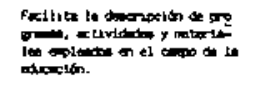 & - - - & 0.000 & 1900 \\
\hline 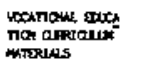 & 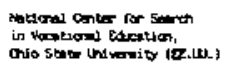 & 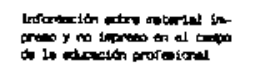 & adm, litros os thato. & 2.30 & 19 \\
\hline
\end{tabular}

